\title{
Harnack inequality for subelliptic $p$-Laplacian equations of Schrödinger type
}

\author{
Yuxing Guo and Yinsheng Jiang*
}

\section{"Correspondence:} ysjiang@xju.edu.cn College of Mathematics and System Sciences, Xinjiang University, Urumqi, Xinjiang 830046, P.R. China

\section{Abstract}

In this paper, we establish the Harnack inequality for weak solutions of nonlinear subelliptic $p$-Laplacian equations of Schrödinger type

$$
-\sum_{k=1}^{m} X_{k}^{*}\left(\langle A(x) X u(x), X u(x)\rangle^{\frac{p-2}{2}} A(x) X_{k} u(x)\right)+V(x)|u(x)|^{p-2} u(x)=f(x)
$$

when the singular potential $V$ is in the Kato-Stummel type class with respect to the Carnot-Carathéodory metric.

MSC: Primary 35J10; secondary 35H2O

Keywords: Harnack inequality; subelliptic $p$-Laplacian; potential; control distance

\section{Introduction}

Let $\Omega$ be a bounded open subset of $\mathbb{R}^{N}$ for $N \geq 3$. Given a family of vector fields $X=$ $\left(X_{1}, \ldots, X_{m}\right)$, we assume that each component $X_{k}=\left(b_{k 1}, \ldots, b_{k N}\right): \Omega \rightarrow \mathbb{R}^{N}$ is locally Lipschitz continuous for $k=1, \ldots, m$. We identify the vector field $X_{k}$ with $X_{k} u=\left\langle X_{k}, \nabla u\right\rangle=$ $\sum_{j=1}^{N} b_{k j} \partial_{j} u$ if $u \in C^{1}(\Omega)$, and when $u \in L_{\text {loc }}^{1}(\Omega)$, it is understood in the distributional sense that

$$
X_{k} u=\sum_{j=1}^{N} \partial_{x_{j}}\left(b_{k j} u\right)-\left(\sum_{j=1}^{N} \partial_{x_{j}}\left(b_{k j}\right)\right) u
$$

for $k=1,2, \ldots, m$.

In this paper, we are interested in nonlinear equations involving the subelliptic $p$-Laplace operator $-\triangle_{p}^{X} u=-\sum_{k=1}^{m} X_{k}^{*}\left(\langle A(x) X u(x), X u(x)\rangle^{\frac{p-2}{2}} A(x) X_{k} u(x)\right)$ and the singular potential $V$ belonging to the Kato-Stummel type class. We consider the nonlinear subelliptic $p$ Laplacian equations of Schrödinger type in $\Omega$

$$
-\sum_{k=1}^{m} X_{k}^{*}\left(\left\langle A(x) X u(x),\left.X u(x)\right|^{\frac{p-2}{2}} A(x) X_{k} u(x)\right)+V(x)|u(x)|^{p-2} u(x)=f(x),\right.
$$

where $p>1, X_{k}^{*}$ denotes the adjoint of $X_{k}$ and each entry of the bounded measurable coefficient matrix $A(x)=\left(a_{i j}(x)\right)$ satisfies $a_{i j}(x) \in L^{\infty}(\Omega)$ and $a_{i j}(x)=a_{j i}(x)$. We suppose that

\section{Springer}

(c) 2013 Guo and Jiang; licensee Springer. This is an Open Access article distributed under the terms of the Creative Commons Attribution License (http://creativecommons.org/licenses/by/2.0), which permits unrestricted use, distribution, and reproduction in any medium, provided the original work is properly cited. 
the operator $-\triangle_{p}^{X}$ satisfies the following $X$-ellipticity condition:

$$
\frac{1}{\lambda} \sum_{k=1}^{m}\left\langle X_{k}(x), \xi\right\rangle^{2} \leq\langle A(x) \xi, \xi\rangle \leq \lambda \sum_{k=1}^{m}\left\langle X_{k}(x), \xi\right\rangle^{2}, \quad \forall x \in \Omega, \forall \xi \in \mathbb{R}^{N},
$$

where $\lambda$ is a positive constant and $\langle\cdot, \cdot\rangle$ denotes the standard inner product in $\mathbb{R}^{N}$. The notion of $X$-ellipticity was implicity introduced in [1] by Franchi and Lanconelli in 1982, after which it was intensively studied in a series of works [2-5], etc. In 2000, it was explicitly developed in [6] by Lanconelli and Kogoj.

For reader's convenience, let us recall the notion of control distance (or the CarnotCarathéodory distance) associated to the family $X$. An absolutely continuous path $\gamma:[0, T] \rightarrow \Omega \subset \mathbb{R}^{N}$ is said to be an $X$-subunit if $\dot{\gamma}(t)=\sum_{k=1}^{m} c_{k}(t) X_{k}(\gamma(t))$, with $\sum_{k=1}^{m} c_{k}^{2}(t) \leq 1$, for almost every $t \in[0, T]$. Assuming that $\Omega \subset \mathbb{R}^{N}$ is $X$-connected, i.e., for every $x, y \in \Omega \subset \mathbb{R}^{N}$, there exists at least one $X$-subunit path connecting $x$ and $y$, we define

$$
d(x, y)=\inf \left\{T>0 \mid \exists \gamma:[0, T] \rightarrow \Omega \subset \mathbb{R}^{N} X \text {-subunit such that } \gamma(0)=x, \gamma(T)=y\right\} .
$$

The mapping $(x, y) \mapsto d(x, y)$ is a metric on $\Omega \subset \mathbb{R}^{N}$. It can be proved that $d(x, y) \rightarrow 0$ implies $|x-y| \rightarrow 0$, where $|\cdot|$ is the Euclidean norm. Hereafter, all the distances mentioned in this context are designated with respect to the metric $d$ unless we indicate it specifically. In particular, $B_{r}(x)$ denotes the ball $\left\{y \in \mathbb{R}^{N} \mid d(x, y)<r\right\}$ with the control metric, and $|E|$ denotes the Lebesgue measure of the set $E \subset \mathbb{R}^{N}$. Following the literature [2], we define the Sobolev spaces for $p>1$ as follows:

$$
W^{1, p}(\Omega, X)=\left\{u \in L^{p}(\Omega) \mid X_{k} u \in L^{p}(\Omega), k=1,2, \ldots, m\right\}
$$

equipped with the norm

$$
\|u\|_{W^{1, p}(\Omega, X)}=\left(\int_{\Omega}\left(|u|^{p}+|X u|^{p}\right) d x\right)^{\frac{1}{p}},
$$

for $X u=\left(X_{1} u, \ldots, X_{m} u\right)$.

Before stating our main results, we present several assumptions of control distance $d(\cdot, \cdot)$ with respect to the vector fields $X$ in $\mathbb{R}^{N}$ and the singular potential $V$ which we will use in the next sections.

(H1) (Metric equivalence) If $|x-y| \rightarrow 0 \Rightarrow d(x, y) \rightarrow 0$, then we have that $|x-y| \rightarrow 0 \Leftrightarrow d(x, y) \rightarrow 0$.

(H2) (Homogeneous dimension) There are positive constants $r_{0}, C_{1}, C_{2}$ and $Q>p>1$ such that for all $r \in\left(0, r_{0}\right]$ and $x \in \Omega$, the following relation is valid:

$$
C_{1} r^{Q} \leq\left|B_{r}(x)\right| \leq C_{2} r^{Q}
$$

where the number $Q$ is chosen as the least integer such that the above inequality holds, which is called the homogeneous dimension of $X$ in $\Omega$.

From the assumptions of control distance $d(\cdot, \cdot)$, the following Sobolev embedding inequality is valid [7]: If $1<p<Q$, then $W_{0}^{1, p}\left(B_{r}, X\right) \hookrightarrow L^{q}\left(B_{r}\right)$ for $1 \leq q \leq \frac{Q p}{Q-p}$. Furthermore, 
there exists a constant $C=C(X, \Omega)>0$, then for all $u \in W_{0}^{1, p}\left(B_{r}, X\right)$,

$$
\left(f_{B_{r}}|u|^{q} d x\right)^{\frac{1}{q}} \leq C r\left(f_{B_{r}}|X u|^{p} d x\right)^{\frac{1}{p}}
$$

According to [8], we have the Poincaré inequality, that is, there is a positive constant $C$ such that the following inequality holds:

$$
f_{B_{r}(x)}\left|u-u_{x, r}\right|^{p} d y \leq C r^{p} f_{B_{\theta r}(x)}|X u|^{p} d y, \quad \forall u \in C^{1}\left(\bar{B}_{\theta r}, X\right),
$$

where $p \geq 1, u_{x, r}=f_{B_{r}} u d x=\frac{1}{\left|B_{r}\right|} \int_{B_{r}} u d x$. We can also refer to [7, 9] for $(p, q)$ type for $1 \leq q \leq \frac{p Q}{Q-p}$.

For the subelliptic $p$-Laplace operator $-\triangle_{p}^{X} u=0$ in $B_{R}$, the Green function $G_{p}(x, y)$ is given by [5], that is, when $x, y \in B_{\frac{R}{4}}$, we have the following decay estimates:

$$
\left|G_{p}(x, y)\right| \leq C \frac{1}{d(x, y)^{(Q-p) /(p-1)}}, \quad 1<p<Q,
$$

where $B_{R}$ is the smallest metric ball such that $\Omega \subset \frac{1}{4} B_{R}$. Also, the Green function estimates for the divergence form of subelliptic operators with nonsmooth coefficients were obtained in [10] for $p=2$.

The assumption on $V$ is that $V \in K_{Q}^{p}(\Omega)$, the Kato-Stummel type class, which means that $V$ is a local integrable function such that $\lim _{r \rightarrow 0^{+}} \eta_{p}(V ; r ; \Omega)=0$, where

$$
\eta_{p}(V ; r ; \Omega)=\sup _{x \in B_{R}} \int_{\{y \in \Omega: d(x, y)<r\}} \frac{|V(y)|}{d(x, y)^{(Q-p) /(p-1)}} d y, \quad 1<p<Q .
$$

Let $\sigma \in \mathbb{R}, M_{\sigma, p}(\Omega)=\left\{V \in L_{\text {loc }}^{1}(\Omega):\|V\|_{\sigma, p, \Omega}<+\infty\right\}$, where

$$
\|V\|_{\sigma, p, \Omega}=\sup _{\substack{x \in \Omega \\ 0<r<\operatorname{diam}(\Omega)}} \frac{1}{r^{\sigma}} \int_{\{y \in \Omega: d(x, y)<r\}} \frac{|V(y)|}{d(x, y)^{(Q-p) /(p-1)}} d y<+\infty .
$$

We remark that if $\sigma>0$ and $1<p<Q$, then $M_{\sigma, p}(\Omega)$ is in fact the Morrey space $L^{1, \mu}(\Omega)$ for some appropriate $\mu$. Moreover, the inclusion $M_{\sigma, p}(\Omega) \subset K_{Q}^{p}(\Omega)$ is trivial.

This article is devoted to presenting the elementary proof of the local estimate and the Harnack inequality for the solutions of Equation (1.1), which is the extension of the related results in $[5,11]$, where $p=2$ or $V=0$, and the Harnack inequality for $p$-subLaplacian in [12].

Our main results are as follows.

Theorem 1.1 Let $u \in W_{\mathrm{loc}}^{1, p}(\Omega, X)$ be a weak solution to Equation (1.1), and let $V, f \in$ $M_{\sigma, p}(\Omega)$ with $0<\sigma<1$ and $1<p<Q$. Assume that $0<r<r_{0}$ with $B_{2 r_{0}} \subseteq \Omega$. Then

$$
\sup _{x \in B_{r}}|u(x)| \leq C\left\{\left(\frac{1}{r^{Q}} \int_{B_{2 r}}|u(x)|^{p} d x\right)^{\frac{1}{p}}+\left(\|f\|_{\sigma, p, B_{2 r}}\right)^{\frac{1}{p-1}}\right\}
$$

with the constant $C>0$ depending only on $Q, p, \lambda$ and $\|V\|_{\sigma, p, B_{2 r_{0}}}$. 
Theorem 1.2 Let $u \in W_{\text {loc }}^{1, p}(\Omega, X)$ be a non-negative weak solution to Equation (1.1) in $\Omega$ and $B_{2 r_{0}} \subset \Omega$, and let $0<r<r_{0}$. Then there exists a constant $C>0$ depending only on $Q$, $p, \lambda$ and $\|V\|_{\sigma, p, B_{2 r_{0}}}$ such that

$$
\sup _{x \in B_{r}} u(x) \leq C\left\{\inf _{x \in B_{r}} u(x)+\left(\|f\|_{\sigma, p, B_{2 r}}\right)^{\frac{1}{p-1}}\right\} .
$$

Corollary 1.1 Under the assumptions of Theorem 1.2, the non-negative weak solution $u \in$ $W_{0}^{1, p}(\Omega, X)$ of Equation (1.1) is locally Hölder continuous in $\Omega$.

We say $u \in W_{\text {loc }}^{1, p}(\Omega)$ is a weak solution of Equation (1.1) if $u$ satisfies

$$
\int_{\Omega}\left\langle A(x) X u,\left.X u\right|^{\frac{p-2}{2}}\langle A(x) X u, X \varphi(x)\rangle d x+\int_{\Omega} V(x)|u|^{p-2} u \varphi(x) d x=\int_{\Omega} f(x) \varphi(x) d x\right.
$$

for any test function $\varphi(x) \in W_{0}^{1, p}(\Omega, X)$.

Throughout this paper, unless otherwise indicated, $C$ is used to denote a positive constant that is not necessarily the same at each occurrence, which depends at most on $Q, p$, $\lambda, V$ and $\Omega$.

\section{Local boundedness of solutions}

The purpose of this section is to show that weak solutions of Equation (1.1) are locally bounded. To do this, we follow the technique by Serrin $[13,14]$.

Lemma 2.1 (Embedding lemma) Let $\Omega$ be an open, bounded and connected set in $\mathbb{R}^{N}$, and suppose $V \in K_{Q}^{p}(\Omega)$ and $1<p<Q$. Then there exists a positive constant $C_{Q, p, \lambda}$ such that for any $u \in W_{0}^{1, p}(\Omega, X)$ and $0<r<\operatorname{diam}(\Omega)$, we have

$$
\int_{\Omega}|V||u|^{p} d x \leq C_{Q, p, \lambda}\left|\eta_{p}(V ; r ; \Omega)\right|^{p-1}\left(\int_{\Omega}|X u|^{p} d x+\frac{1}{r^{p}} \int_{\Omega}|u|^{p} d x\right) .
$$

Proof Let $G_{p}(x, y)$ be the Green function for $-\triangle_{p}^{X}$ in $B_{r} \cap \Omega$. We set

$$
\psi(x)=\int_{B_{r} \cap \Omega} V(y) G_{p}(x, y) d y .
$$

This is well defined, and $\|\psi\|_{L^{\infty}} \leq C \eta_{p}(V ; r ; \Omega)$ since $V \in K_{Q}^{p}(\Omega)$. Moreover, $\psi(x)$ is the weak solution to the equation $-\triangle_{p}^{X} \psi=V$ in $B_{r} \cap \Omega$, and also $\psi(x)=0$ on $\partial\left(B_{r} \cap \Omega\right)$.

For $p>1$ and $a, b, \varepsilon>0$, it is easy to see from the Young inequality that

$$
\begin{aligned}
& p a^{p-1} b \leq(p-1) \varepsilon^{-1 /(p-1)} a^{p}+\varepsilon b^{p}, \\
& p a^{p-1} b \leq \varepsilon a^{p}+(p-1)^{p-1} \varepsilon^{-1 /(p-1)} b^{p} .
\end{aligned}
$$

From the uniform $X$-ellipticity assumption (1.2), a direct calculation shows

$$
\frac{1}{\lambda}|X \varphi|^{2} \leq\langle A(x) \nabla \varphi, \nabla \varphi\rangle \leq \lambda|X \varphi|^{2}, \quad|\langle A(x) \nabla \varphi, \nabla \psi\rangle| \leq \lambda|X \varphi||X \psi|,
$$


when $\varphi$ and $\psi \in C^{1}(\Omega)$. Assume that $u \in W_{0}^{1, p}\left(B_{r} \cap \Omega, X\right)$, then by the inequality (2.1), we get that

$$
\begin{aligned}
\int_{B_{r} \cap \Omega} V|u|^{p} d x & =-\int_{B_{r} \cap \Omega}|u|^{p} \triangle_{p}^{X} \psi d x \\
& \leq \lambda^{\frac{p}{2}} p \int_{B_{r} \cap \Omega}|X \psi|^{p-1}|u|^{p-1}|X u| d x \\
& \leq \lambda^{\frac{p}{2}} \frac{\varepsilon}{4} \int_{B_{r} \cap \Omega}|X u|^{p} d x+\lambda^{\frac{p}{2}}(p-1)\left(\frac{\varepsilon}{4}\right)^{-1 /(p-1)} \int_{B_{r} \cap \Omega}|X \psi|^{p}|u|^{p} d x
\end{aligned}
$$

for any $\varepsilon>0$. In addition, we note that

$$
\begin{aligned}
\int_{B_{r} \cap \Omega} V|u|^{p} \psi d x= & \int_{B_{r} \cap \Omega}\langle A(x) X \psi, X \psi\rangle^{\frac{p-2}{2}}\langle A(x) X \psi, X \psi\rangle|u|^{p} d x \\
& +p \int_{B_{r} \cap \Omega}\langle A(x) X \psi, X \psi\rangle^{\frac{p-2}{2}}\langle A(x) X \psi, X u\rangle \psi|u|^{p-1} \frac{u}{|u|} d x .
\end{aligned}
$$

This and the inequality (2.2) with $\varepsilon=1 /\left(2 \lambda^{p}\right)$ yield that

$$
\begin{aligned}
\int_{B_{r} \cap \Omega}|X \psi|^{p}|u|^{p} d x \leq & \lambda^{\frac{p}{2}} \int_{B_{r} \cap \Omega}|A(x) X \psi, X \psi\rangle^{\frac{p-2}{2}}\langle A(x) X \psi, X \psi\rangle|u|^{p} d x \\
\leq & \lambda^{\frac{p}{2}} \int_{B_{r} \cap \Omega}|V||u|^{p} \psi d x+\lambda^{p} p \int_{B_{r} \cap \Omega}|X \psi|^{p-1}|u|^{p-1}|X u||\psi| d x \\
\leq & \lambda^{\frac{p}{2}} \int_{B_{r} \cap \Omega}|V||u|^{p} \psi d x+\lambda^{\frac{p^{2}}{p-1}}(2(p-1))^{p-1} \int_{B_{r} \cap \Omega}|X u|^{p}|\psi|^{p} d x \\
& +\frac{1}{2} \int_{B_{r} \cap \Omega}|X \psi|^{p}|u|^{p} d x .
\end{aligned}
$$

From the inequalities (2.3) and (2.4), and the estimation of $\psi$, we obtain that

$$
\begin{aligned}
\int_{B_{r} \cap \Omega} V|u|^{p} d x \leq & \lambda^{\frac{p}{2}} \frac{\varepsilon}{4} \int_{B_{r} \cap \Omega}|X u|^{p} d x+C_{Q, p, \lambda}\left(\frac{\varepsilon}{4}\right)^{-1 /(p-1)} \eta_{p}(V ; r ; \Omega) \int_{B_{r} \cap \Omega}|V||u|^{p} d x \\
& +C_{Q, p, \lambda}\left(\frac{\varepsilon}{4}\right)^{-1 /(p-1)} \eta_{p}(V ; r ; \Omega)^{p} \int_{B_{r} \cap \Omega}|X u|^{p} d x .
\end{aligned}
$$

To complete the proof of the lemma, we employ the method of the proof of Lemma 3.3 in [15]. Given $0<\delta<1$, let $\left\{\psi_{j}^{p}\right\}_{1}^{l}$ be a finite partition of unity of $\bar{\Omega}$ such that $\operatorname{supp} \psi_{j} \subseteq B_{r_{j}}\left(x_{j}\right)$ with $x_{j} \in \bar{\Omega}$ and $0<r_{j} \leq \delta$. Set $\Omega^{*}=\left\{x: d(x, \bar{\Omega}) \leq \varepsilon_{0}\right\} \subset \frac{1}{4} B_{R}$ and further restrict $\delta<\varepsilon_{0}$. Therefore,

$$
\begin{aligned}
\int_{\Omega} V\left|u \psi_{j}\right|^{p} d x \leq & \lambda^{\frac{p}{2}} \frac{\varepsilon}{4} \int_{\Omega}\left|X\left(u \psi_{j}\right)\right|^{p} d x+C_{Q, p, \lambda}\left(\frac{\varepsilon}{4}\right)^{-1 /(p-1)} \eta_{p}(V ; \delta ; \Omega) \int_{\Omega} V\left|u \psi_{j}\right|^{p} d x \\
& +C_{Q, p, \lambda}\left(\frac{\varepsilon}{4}\right)^{-1 /(p-1)} \eta_{p}(V ; \delta ; \Omega)^{p} \int_{\Omega}\left|X\left(u \psi_{j}\right)\right|^{p} d x .
\end{aligned}
$$

We now choose $\varepsilon$ such that

$$
C_{Q, p, \lambda} \eta_{p}(V ; \delta ; \Omega)=\frac{1}{2}\left(\frac{\varepsilon}{4}\right)^{1 /(p-1)}, \quad C_{Q, p, \lambda} \eta_{p}(V ; \delta ; \Omega)^{p}=\left(\frac{\varepsilon}{4}\right)^{1 /(p-1)} \lambda^{\frac{p}{2}} \frac{3 \varepsilon}{4},
$$


which implies that

$$
\begin{aligned}
\int_{\Omega} V\left|u \psi_{j}\right|^{p} d x & \leq \lambda^{\frac{p}{2}} \varepsilon \int_{\Omega}\left|X\left(u \psi_{j}\right)\right|^{p} d x \\
& \leq \lambda^{\frac{p}{2}} 2^{p-1} \varepsilon\left(\int_{\Omega}|X u|^{p}\left|\psi_{j}\right|^{p} d x+\int_{\Omega}\left|X \psi_{j}\right|^{p}|u|^{p} d x\right) .
\end{aligned}
$$

By summing in $j$, it follows that

$$
\begin{aligned}
\int_{\Omega} V|u|^{p} d x & \leq \lambda^{\frac{p}{2}} 2^{p-1} \varepsilon\left(\left.\int_{\Omega}|X u|^{p}\left|d x+\sum_{j=1}^{l} \frac{l(\varepsilon)}{\delta^{p}} \int_{\Omega}\right| u\right|^{p} d x\right) \\
& \leq C_{Q, p, \lambda}\left|\eta_{p}(V ; \delta ; \Omega)\right|^{p-1}\left(\int_{\Omega}|X u|^{p} d x+\frac{1}{\delta^{p}} \int_{\Omega}|u|^{p} d x\right)
\end{aligned}
$$

with the constant $C_{Q, p, \lambda}>0$. It is clear that the inequality (2.6) implies the desired conclusion.

Lemma 2.2 Let $\Omega$ be an open, bounded and connected set in $\mathbb{R}^{N}$, and suppose $V \in M_{\sigma, p}(\Omega)$ with $0<\sigma<1$ and $1<p<Q$. Then, for any $0<\varepsilon<1$, there exists a constant $\tau>0$ such that for any $u \in W_{0}^{1, p}(\Omega, X)$, we have

$$
\int_{\Omega} V|u|^{p} d x \leq \varepsilon \int_{\Omega}|X u|^{p} d x+C \varepsilon^{-\tau} \int_{\Omega}|u|^{p} d x
$$

Proof Repeating the proof of Lemma 2.1 and noting $\eta(V ; \delta ; \Omega) \leq\|V\|_{\sigma, p} \delta^{\sigma}$, we can deduce from the inequality (2.6) that the lemma holds with $\tau=\frac{p}{(p-1) \sigma}-1$ and $C=$ $C_{Q, p, \lambda, \sigma}\left(\|V\|_{\sigma, p}\right)^{p / \sigma}$ as long as we choose

$$
\delta=\left(\frac{\varepsilon}{C_{Q, p, \lambda}\|V\|_{\sigma, p}^{p-1}}\right)^{\frac{1}{(p-1) \sigma}} .
$$

We are ready to show the local maximal estimates, i.e., Theorem 1.1.

Proof of Theorem 1.1 For $q \geq 1$ and $h \leq M<\infty$, where $h$ is a positive number which will be determined later, we define the function $F_{M}: \mathbb{R}^{+} \rightarrow \mathbb{R}^{+}$by

$$
F_{M}(t)= \begin{cases}t^{q} & \text { if } 0 \leq t \leq M, \\ q M^{q-1}(t-M)+M^{q} & \text { if } t>M .\end{cases}
$$

Note that $F_{M}^{\prime}(t)$ is non-decreasing, non-negative and bounded for each fixed $M$, and $t F_{M}^{\prime}(t) \leq q F_{M}(t)$ for any $t \geq 0$. Let $u \in W_{\text {loc }}^{1, p}(\Omega, X)$ be the weak solution to Equation (1.1), and let $v=|u|+h$. We consider the function $G_{M}(u)=\operatorname{sign}(u) \int_{0}^{v}\left(F_{M}^{\prime}(t)\right)^{p} d t$. It is easy to see that

$$
G_{M}^{\prime}(u)=\left(F_{M}^{\prime}(v)\right)^{p} \quad \text { and } \quad\left|G_{M}(u)\right| \leq\left(F_{M}^{\prime}(v)\right)^{p-1} F_{M}(v)
$$

for any $-\infty<u<\infty$. Moreover, $G_{M}(u) \in W_{\mathrm{loc}}^{1, p}(\Omega, X)$. 
Thus, for any non-negative function $\phi(x) \in C_{0}^{\infty}(\Omega)$, we can choose $\psi(x)=\phi^{p}(x) G_{M}(u(x))$ as a testing function in Equation (1.1), which yields

$$
\begin{gathered}
\int_{\Omega}\left\langle A(x) X u,\left.X u\right|^{\frac{p-2}{2}}\left\langle A(x) X u,\left(p \phi^{p-1} G_{M}(u) X \phi+\phi^{p} G_{M}^{\prime}(u) X u\right)\right\rangle d x\right. \\
=-\int_{\Omega} V(x)|u(x)|^{p-2} u(x) \phi^{p} G_{M}(u) d x+\int_{\Omega} f(x) \phi^{p} G_{M}(u) d x .
\end{gathered}
$$

Thus

$$
\begin{aligned}
\int_{\Omega} \phi^{p} G_{M}^{\prime}(u)|X u|^{p} d x \leq & \lambda^{\frac{p}{2}} \int_{\Omega}\left\langle A(x) X u,\left.X u\right|^{\frac{p-2}{2}}\left\langle A(x) X u, \phi^{p} G_{M}^{\prime}(u) X u\right\rangle d x\right. \\
\leq & \lambda^{p} p \int_{\Omega}|X u|^{p-1} \phi^{p-1}\left|G_{M}(u)\right||X \phi| d x \\
& +\lambda^{\frac{p}{2}} \int_{\Omega}|V||u|^{p-1} \phi^{p}\left|G_{M}(u)\right| d x+\lambda^{\frac{p}{2}} \int_{\Omega}|f(x)| \phi^{p}\left|G_{M}(u)\right| d x .
\end{aligned}
$$

Recalling that $v=|u|+h$ and so $|X v|=|X u|$, using the estimates (2.7) and the fact that $F_{M}^{\prime}(v) \leq q F_{M}(v) / v \leq h^{-1} q F_{M}(v)$, we can then obtain that

$$
\begin{aligned}
\int_{\Omega}|X v|^{p} \phi^{p}\left(F_{M}^{\prime}(v)\right)^{p} d x \leq & \lambda^{p} p \int_{\Omega}|X v|^{p-1} \phi^{p-1} F_{M}(v)\left(F_{M}^{\prime}(v)\right)^{p-1}|X \phi| d x \\
& +\lambda^{\frac{p}{2}} q^{p-1} \int_{\Omega}|V(x)| \phi^{p}\left|F_{M}(v)\right|^{p} d x \\
& +\lambda^{\frac{p}{2}} h^{1-p} q^{p-1} \int_{\Omega}|f(x)| \phi^{p}\left|F_{M}(v)\right|^{p} d x .
\end{aligned}
$$

Using the inequality $(2.2)$ with $\varepsilon=1 /\left(2 \lambda^{p}\right)$, we have

$$
\int_{\Omega}|X v|^{p} \phi^{p}\left(F_{M}^{\prime}(v)\right)^{p} d x \leq \lambda^{\frac{p^{2}}{p-1}} 2^{p}(p-1)^{p-1} \int_{\Omega}|X \phi|^{p} F_{M}^{p} d x+\lambda^{\frac{p}{2}} 2 q^{p-1} \int_{\Omega} W \phi^{p} F_{M}^{p} d x
$$

where $W(x)=|V(x)|+h^{1-p}|f(x)|$. We set $U(x)=F_{M}(v(x))$ and assume supp $\phi \subset B_{2 r}$, then we rewrite the inequality above as follows:

$$
\int_{B_{2 r}}|X U|^{p} \phi^{p} d x \leq \lambda^{\frac{p^{2}}{p-1}} 2^{p}(p-1)^{p-1} \int_{B_{2 r}}|X \phi|^{p} U^{p} d x+\lambda^{\frac{p}{2}} 2 q^{p-1} \int_{B_{2 r}} W \phi^{p} U^{p} d x
$$

Hence

$$
\int_{B_{2 r}}|X(U \phi)|^{p} d x \leq \lambda^{\frac{p^{2}}{p-1}} 4^{p} p^{p-1} \int_{B_{2 r}}|X \phi|^{p} U^{p} d x+\lambda^{\frac{p}{2}} 2^{p} q^{p-1} \int_{B_{2 r}} W \phi^{p} U^{p} d x
$$

Now we take $h=\left(\|f\|_{\sigma, p, B_{2 r}}\right)^{1 /(p-1)}$, and so $\|W\|_{\sigma, p, B_{2 r}} \leq\|V\|_{\sigma, p, B_{2 r}}+1$. By Lemma 2.2 we have, for any $\varepsilon>0$,

$$
\int_{B_{2 r}} W \phi^{p} U^{p} d x \leq \varepsilon \int_{B_{2 r}}|X(U \phi)|^{p} d x+C \varepsilon^{-\tau} \int_{B_{2 r}} \phi^{p} U^{p} d x
$$


with the positive constants $C$ and $\tau$ independent of $f, u$ and $\phi$. From this and the inequality (2.8) it follows that

$$
\int_{B_{2 r}}|X(U \phi)|^{p} d x \leq C \int_{B_{2 r}}|X \phi|^{p} U^{p} d x+C q^{(p-1)(\tau+1)} \int_{B_{2 r}} \phi^{p} U^{p} d x
$$

where $C$ is a positive constant depending on $Q, p$ and $\lambda$.

By the Sobolev embedding inequality, we get from (2.9) that

$$
\left(\int_{B_{2 r}}|U \phi|^{p \kappa} d x\right)^{1 /(p \kappa)} \leq C\left\{\int_{B_{2 r}}|X \phi|^{p} U^{p} d x+q^{(p-1)(\tau+1)} \int_{B_{2 r}} \phi^{p} U^{p} d x\right\}^{1 / p}
$$

with absolute constant $C$, where $\kappa=\frac{Q}{Q-p}>1$.

Let $r_{1}$ and $r_{2}$ be such that $r \leq r_{1}<r_{2} \leq 2 r$, taking $\phi(x)$ in such a way that $\phi(x)=1$ in $B_{r_{1}}$, $0 \leq \phi(x) \leq 1$ in $B_{r_{2}}$ and $|X \phi(x)| \leq \frac{C}{r_{2}-r_{1}}$, and recalling $U=F_{M}(v)$ and $q \geq 1$, we thus obtain

$$
\left(\int_{B_{r_{1}}}\left|F_{M}(v)\right|^{p \kappa} d x\right)^{1 /(p \kappa)} \leq \frac{C q^{1+\tau}}{r_{2}-r_{1}}\left(\int_{B_{r_{2}}}\left|F_{M}(v)\right|^{p} d x\right)^{1 / p}
$$

Letting $M \rightarrow+\infty$, then

$$
\left(\int_{B_{r_{1}}}|\nu|^{p q \kappa} d x\right)^{1 /(p q \kappa)} \leq\left(\frac{C q^{1+\tau}}{r_{2}-r_{1}}\right)^{1 / q}\left(\int_{B_{r_{2}}}|\nu|^{p q} d x\right)^{1 /(p q)}
$$

with the constant $C$ independent of $r_{1}, r_{2}, q$ and $v$.

By the standard iteration argument [14], we set $\theta_{i}=p \kappa^{i}$ and $r_{i}=r\left(1+2^{-i}\right)$ for $i=0,1,2, \ldots$. Hence the previous inequality $(2.10)$ becomes

$$
\|v\|_{L^{\theta_{i}+1}\left(B_{r_{i}+1}\right)} \leq C^{\frac{p}{\theta_{i}}}\left(\frac{2^{i+1}}{r}\right)^{\frac{p}{\theta_{i}}}\left(\frac{\theta_{i}}{p}\right)^{\frac{p}{\theta_{i}}(1+\tau)}\|v\|_{L^{\theta_{i}}\left(B_{r_{i}}\right)} .
$$

Iteration yields

$$
\begin{aligned}
\|v\|_{L^{\infty}\left(B_{r}\right)} & \leq\left(\frac{C}{r}\right)^{\sum_{i=0}^{\infty} \frac{1}{\kappa^{i}}} \prod_{i=0}^{\infty}\left(2^{i+1}\right)^{\frac{1}{\kappa^{i}}} \prod_{i=0}^{\infty}\left(\kappa^{i}\right)^{\frac{1+\tau}{\kappa^{i}}}\|v\|_{L^{p}\left(B_{2 r}\right)} \\
& \leq C r^{-\frac{Q}{p}}\|v\|_{L^{p}\left(B_{2 r}\right)} \leq C\left(\frac{1}{\left|B_{2 r}\right|} \int_{B_{2 r}}|\nu|^{p} d x\right)^{\frac{1}{p}}
\end{aligned}
$$

with the constant $C$ independent of $v$. Recalling $v=|u|+h$ and that $h=\|f\|_{\sigma, p, B_{2 r}}^{1 /(p-1)}$, we get the conclusion. This completes the proof of the theorem.

\section{Harnack inequality of solutions}

In this section we give the proof of Theorem 1.2, i.e., the Harnack inequality.

Proof of Theorem 1.2 Proceeding as in the proof of Theorem 1.1, it is sufficient to prove the theorem by assuming $r=1$. The general case $r \neq 1$ follows by dilations. We set $v=|u|+h$ with $h=\left(\|f\|_{\sigma, p, B_{3}}\right)^{1 /(p-1)}$ and $W=V+h^{1-p} f$, and take $\psi(x)=\phi^{p} \nu^{\beta}(x)$ as a test function in 
the inequality (1.3), where $\phi(x)$ is a non-negative smooth function such that $\operatorname{supp} \phi(x) \subseteq B_{3}$ and $\beta \in \mathbb{R}$. We obtain

$$
\begin{aligned}
\lambda^{-\frac{p}{2}}|\beta| \int_{\Omega}|X \nu|^{p} \nu^{\beta-1} \phi^{p} d x \leq & \lambda^{\frac{p}{2}} p \int_{\Omega}|X \nu|^{p-1}|X \phi| \phi^{p-1} \nu^{\beta} d x \\
& +\int_{\Omega}|V(x)||\nu|^{p-1+\beta} \phi^{p} d x+\int_{\Omega}|f(x)||\nu|^{\beta} \phi^{p} d x .
\end{aligned}
$$

Then it follows from the Cauchy inequality that

$$
\int_{\Omega}|X \nu|^{p} \nu^{\beta-1} \phi^{p} d x \leq C(\lambda) \frac{p^{p}}{|\beta|^{p}} \int_{\Omega}|X \phi|^{p}|\nu|^{p-1+\beta} d x+C(\lambda) \frac{p}{|\beta|} \int_{\Omega} W \phi^{p}|\nu|^{p-1+\beta} d x .
$$

We set

$$
U(x)= \begin{cases}v(x)^{\frac{p-1+\beta}{p}} & \text { if } \beta \neq 1-p \\ \log v(x) & \text { if } \beta=1-p .\end{cases}
$$

Then, from the above inequality, in the case $\beta \neq 1-p$, we see that

$$
\frac{p^{p}}{(p-1+|\beta|)^{p}} \int_{\Omega}|X U|^{p} \phi^{p} d x \leq C(\lambda) \frac{p^{p}}{|\beta|^{p}} \int_{\Omega}|X \phi|^{p} U^{p} d x+C(\lambda) \frac{p}{|\beta|} \int_{\Omega} W \phi^{p} U^{p} d x
$$

i.e.,

$$
\int_{\Omega}|X U|^{p} \phi^{p} d x \leq C_{1}(\lambda, \beta) \int_{\Omega}|X \phi|^{p} U^{p} d x+C_{2}(\lambda, \beta) \int_{\Omega} W \phi^{p} U^{p} d x
$$

where $C_{1}(\lambda, \beta)=\frac{\left(p-1+|\beta|^{p}\right.}{|\beta|^{p}} C(\lambda)$ and $C_{2}(\lambda, \beta)=\frac{(p-1+|\beta|)^{p}}{|\beta|} C(\lambda)$. Simultaneously, in the case $\beta=1-p$, we can see that

$$
\int_{\Omega}|X U|^{p} \phi^{p} d x \leq C(\lambda) 2^{p} \int_{\Omega}|X \phi|^{p} d x+C(\lambda) 2 \int_{\Omega} W \phi^{p} d x .
$$

To our destination, we exploit the inequality (3.2) and use Lemma 2.1 with $r=3$ to get

$$
\int_{\Omega}|X U|^{p} \phi^{p} d x \leq C 2^{p+1}\left\{\int_{\Omega}|X \phi|^{p} d x+\int_{\Omega} \phi^{p} d x\right\}
$$

where $C$ is a positive constant depending only on $Q, p, \lambda$ and $\|V\|_{\sigma, p, B_{3}}$. Choosing $\phi(x)$ in such a way that $\phi(x)=1$ in $B_{\rho}$, supp $\phi \subset B_{2 \rho} \subset B_{3}$, and $|X \phi(x)| \leq \frac{C}{\rho}$, where $B_{\rho}$ is an arbitrary open ball contained in $B_{2}$, we then obtain that

$$
\left(\frac{1}{\left|B_{\rho}\right|} \int_{B_{\rho}}|X U|^{p} d x\right)^{\frac{1}{p}} \leq C \frac{1}{\rho} .
$$

Thus, by the assumption of (H3) Poincaré inequality, we get that $U$ is a BMO function, and 
so the John-Nirenberg lemma for BMO function yields that there exist positive constants $q_{0}$ and $C$ such that

$$
\left(\frac{1}{\left|B_{2}\right|} \int_{B_{2}} e^{q_{0} U} d x\right)^{\frac{1}{q_{0}}}\left(\frac{1}{\left|B_{2}\right|} \int_{B_{2}} e^{-q_{0} U} d x\right)^{\frac{1}{q_{0}}} \leq C
$$

Set

$$
\Phi(q, r)=\left(\frac{1}{\left|B_{r}\right|} \int_{B_{r}} v^{q} d x\right)^{\frac{1}{q}}
$$

for any real number $q \neq 0$ and $r>0$. Then from the previous inequality, recalling that $U=\log v$, we have

$$
\Phi\left(q_{0}, 2\right) \leq C \Phi\left(-q_{0}, 2\right)
$$

Now we turn our attention to (3.1). By Lemma 2.2 we obtain

$$
\begin{aligned}
\int_{\Omega}|X(U \phi)|^{p} d x \leq & C C_{1}(\lambda, \beta) \int_{\Omega}|X \phi|^{p} U^{p} d x \\
& +C C_{2}(\lambda, \beta) \varepsilon \int_{\Omega}|X(U \phi)|^{p} d x+C C_{2}(\lambda, \beta) \varepsilon^{-\tau} \int_{\Omega} \phi^{p} U^{p} d x
\end{aligned}
$$

with the constant $C>0$ depending only $Q$ and $p$. We choose $\varepsilon$ such that $C C_{2}(\lambda, \beta) \varepsilon=1 / 2$, and so we deduce that

$$
\int_{\Omega}|X(U \phi)|^{p} d x \leq C C_{1}(\lambda, \beta) \int_{\Omega}|X \phi|^{p} U^{p} d x+C\left(C_{2}(\lambda, \beta)\right)^{\tau+1} \int_{\Omega} \phi^{p} U^{p} d x
$$

Let $r_{1}$ and $r_{2}$ be such that $0<r_{1}<r_{2} \leq 2$, choosing $\phi(x)$ in such a way that $\phi(x)=1$ in $B_{r_{1}}$, $0 \leq \phi(x) \leq 1$ in $B_{r_{2}}$ and $|X \phi(x)| \leq \frac{C}{r_{2}-r_{1}}$. From (3.4) and the Sobolev embedding inequality, we obtain

$$
\begin{aligned}
\left(\int_{B_{r_{1}}}|U|^{p \kappa} d x\right)^{\frac{1}{\kappa}} & \leq C\left\{\frac{C_{1}(\lambda, \beta)}{\left(r_{2}-r_{1}\right)^{p}}+\left(C_{2}(\lambda, \beta)\right)^{\tau+1}\right\} \int_{B_{r_{2}}} U^{p} d x \\
& \leq \frac{C_{Q, p, \tau}}{\left(r_{2}-r_{1}\right)^{p}}\left(1+\frac{p-1}{|\beta|}\right)^{p+1+\tau}\left(1+\frac{|\beta|}{p-1}\right)^{(p-1)(\tau+1)} \int_{B_{r_{2}}} U^{p} d x
\end{aligned}
$$

with $\kappa=\frac{Q}{Q-p}>1$ and the constant $C_{Q, p, \tau}$ depending only on $Q, p$ and $\tau$.

Put $q=p-1+\beta \neq p-1$ and take the $q$ th root of each side of (3.5) for positive $q$ and negative $q$, respectively. Then by the iteration arguments used in the proof of Theorem 1.1, we choose the initial index $q=q_{0}^{\prime}$ with some $0<q_{0}^{\prime} \leq q_{0}$, if $q>0$, such that $p-1$ lies midway between two consecutive iterates of $\kappa^{i} q_{0}^{\prime}$ for $i=1,2, \ldots$, which certifies that $|\beta| \geq \frac{\kappa-1}{\kappa+1}(p-1)$, and we choose the initial index $q=-q_{0}$ if $q<0$,

$$
\sup _{x \in B_{1}}|v(x)| \leq C \Phi\left(q_{0}^{\prime}, 2\right)
$$


and

$$
\inf _{x \in B_{1}}|v(x)| \geq C \Phi\left(-q_{0}, 2\right)
$$

Therefore, from (3.3), (3.6), (3.7) and the Hölder inequality, we have arrived at

$$
\sup _{x \in B_{1}}|v(x)| \leq C \inf _{x \in B_{1}}|v(x)|
$$

which implies the desired conclusion of Theorem 1.2.

\section{Competing interests}

The authors declare that they have no competing interests.

\section{Authors' contributions}

The authors did not provide this information.

\section{Acknowledgements}

The second author is supported by the NSF of China (No. 11161044).

\section{Received: 14 May 2012 Accepted: 14 March 2013 Published: 8 April 2013}

\section{References}

1. Franchi, B, Lanconelli, E: Une métrique associée à une class d'opérateurs elliptiques dégénérés. In: Conference on Linear Partial and Pseudo-Differential Operators, Torino, 1982, pp. 105-114 (1984) (in French). Special issue on Rend. Sem. Mat. Univ. Politec. Torino 1983

2. Capogna, L, Danielli, D, Garofalo, N: An embedding theorem and the Harnack inequality for nonlinear subelliptic equations. Commun. Partial Differ. Equ. 18(9-10), 1765-1794 (1993)

3. Fazio, GD, Zamboni, P: Unique continuation of non negative solutions to quasilinear subelliptic equations in Carnot Carathéodory spaces. Commun. Appl. Nonlinear Anal. 10(2), 97-105 (2003)

4. Mazzoni, G: Green function for X-elliptic operators. Manuscr. Math. 115(2), 207-238 (2004)

5. Zheng, S, Feng, Z: Green functions for a class of nonlinear degenerate operators with X-ellipticity. Trans. Am. Math. Soc. 364(7), 3627-3655 (2012)

6. Lanconelli, E, Kogoj, AE: $X$-elliptic operators and X-control distances, contributions in honor of the memory of Ennio De Giorgi. Ric. Mat. 49(suppl): 223-243 (2000)

7. Lu, G: Weighted Poincaré and Sobolev inequalities for vector fields satisfying Hörmander's condition and applications. Rev. Mat. Iberoam. 8(3), 367-439 (1992)

8. Jerison, D: The Poincaré inequality for vector fields satisfying Hörmander's condition. Duke Math. J. 53(2), 503-523 (1986)

9. Lu, G: The sharp Poincaré inequality for free vector fields: an endpoint result. Rev. Mat. Iberoam. 10(2), 453-466 (1994)

10. Lu, G: Existence and size estimates for the Green's functions of differential operators constructed from degenerate vector fields. Commun. Partial Differ. Equ. 17(7-8), 1213-1251 (1992)

11. Lu, G: On Harnack's inequality for a class of strongly degenerate Schrödinger operators formed by vector fields. Differ. Integral Equ. 7(1), 73-100 (1994)

12. Lu, G: Embedding theorems into Lipschitz and BMO spaces and applications to quasilinear subelliptic differential equations. Publ. Mat. 40(2), 301-329 (1996)

13. Serrin, J: Local behavior of solutions of quasilinear equations. Acta Math. 111(1), 247-302 (1964)

14. Zamboni, P: Hölder continuity for solutions of linear degenerate elliptic equations under minimal assumptions. J. Differ. Equ. 182(1), 121-140 (2002)

15. Grutierrez, C: Harnack's inequality for degenerate Schrödinger operators. Trans. Am. Math. Soc. 312(1), 403-419 (1989)

doi:10.1186/1029-242X-2013-160

Cite this article as: Guo and Jiang: Harnack inequality for subelliptic $p$-Laplacian equations of Schrödinger type.

Journal of Inequalities and Applications 2013 2013:160. 